\title{
Aislamiento y caracterización sintomática de Pyricularia spp. en arroz y otros hospedantes en la provincia de Corrientes (Argentina).
}

\author{
Lisandro Martín Bastida $^{1} \oplus$, Susana Alejandra Gutierrez ${ }^{1} \oplus$ Marcelo Aníbal Carmona $^{2}$
}

\begin{abstract}
${ }^{1}$ Universidad Nacional del Nordeste, CONICET, Facultad de Ciencias Agrarias, Cátedra de Fitopatología, Sargento Cabral 2131, CP 3400 Corrientes, Argentina. ${ }^{2}$ Universidad de Buenos Aires, Facultad de Agronomía, Cátedra de Fitopatología, Av. San Martin 4453, C1417DSE - Buenos Aires, Argentina.

Autor para correspondencia: Lisandro Martín Bastida (lisandrobastida@hotmail.com)

Data de chegada: 31/01/2018. Aceito para publicação em: 26/11/2018.
\end{abstract}

$10.1590 / 0100-5405 / 190957$

\section{RESUMEN}

Bastida, L.M.; Gutierrez, S.A.; Carmona, M.A. Aislamiento y caracterización sintomática de Pyricularia spp. en arroz y otros hospedantes en la provincia de Corrientes (Argentina). Summa Phytopathologica, v.45, n.2, p.200-203, 2019.

Pyricularia spp. es un complejo fúngico causante de la enfermedad conocida como tizón en más de 50 especies de la familia Poaceae. El más conocido es Pyricularia oryzae Cavara, patógeno en cultivos principales como arroz, trigo, mijo, cebada y malezas. Con el objetivo de actualizar el rango de hospedantes, diferenciar la sintomatología, aislar las cepas de la región y caracterizarlas a través del estudio morfométrico y cultural, se realizó durante la campaña 2016/17, el relevamiento de plantas con síntomas de tizón en campos de la provincia de Corrientes. El agente causal fue identificado por caracterización morfométrica y cultural como Pyricularia spp.

Palabras clave: micosis, tizón, Poáceas

\section{RESUMO}

Bastida, L.M.; Gutierrez, S.A.; Carmona, M.A. Isolamento e caracterização sintomática de Pyricularia spp. em arroz e outros hospedeiros na província de Corrientes (Argentina). Summa Phytopathologica, v.45, n.2, p.200-203, 2019.

Pyricularia spp. é um complexo fúngico causamte da doença conhecida como brusone em mais de 50 espécies da família Poaceae. O mais conhecido é Pyricularia oryzae Cavara, patógeno em grandes culturas como arroz, trigo, milheto, cevada e plantas daninhas. Com o objetivo de atualizar a gama de hospedeiros, diferenciar os sintomas, isolar as cepas da região e caracterizá-las através do estudo morfométrico e cultural, foi realizado durante a campanha 2016/17, o levantamento de plantas com sintomas da doença em campos da província de Corrientes. O agente causal foi identificado por caracterização morfométrica e cultural como Pyricularia spp.

Palavras chave: micose, brusone, Poaceae

Pyricularia spp. es un complejo fúngico $(2,12)$, el más conocido es Pyricularia oryzae Cavara (teleomorfo Magnaporthe oryzae B. Couch) agente causal de la enfermedad conocida como tizón del arroz (Oryza sativa), considerada como la más difundida y destructiva a nivel mundial $(7,11,12)$.

En Corrientes el patógeno es endémico afectando principalmente al arroz, no obstante también fue reportado en trigo, cebada forrajera, setaria, ray-grass, alpiste y más de 50 hospedantes, entre plantas cultivadas y espontáneas principalmente de la familia Poaceae, muchas de las cuales son malezas comunes en el cultivo de arroz $(1,3,4,5$, $7,8,9,10,13)$.

Con el objetivo de actualizar el rango de hospedantes, aislar las cepas de la región y caracterizarlas a través del estudio morfométrico y cultural, se realizó durante la campaña 2016/17 el relevamiento de plantas con síntomas de tizón en cultivos de arroz en los departamentos de Lavalle, Ita Ibate, Berón de Astrada, Curuzú Cuatiá, Empedrado, Goya, Mercedes, San Roque y Capital de la provincia de Corrientes, Argentina.

Se seleccionaron muestras con síntomas de tizón y se analizaron en el Laboratorio de la Cátedra de Fitopatología de la Facultad de Ciencias Agrarias de la Universidad Nacional del Nordeste (FCAUNNE). Las características morfométricas se determinaron midiendo conidios y conidióforos obtenidos a partir de las lesiones, utilizando un microscopio ENOSA 40x con ocular micrométrico. Las características culturales se determinaron en agar papa glucosado (APG) al 1,5\% pH 6 .

Para la obtención de aislados de Pyricularia spp. se efectuaron siembras comunes de trocitos de hojas tomadas del límite entre tejido sano y enfermo, los que se desinfectaron en hipoclorito de sodio al $2,5 \%$ durante un minuto, transfiriéndolos a cajas de Petri con APG al $1,5 \%, \mathrm{pH}$ 6. Las colonias se desarrollaron en campana de iluminación $12 \mathrm{~h}$ luz - 12 h oscuridad por 8 días (lámpara Philips TL-D 36W/54$7651 \mathrm{SL} / 25)$ a $25 \pm 2{ }^{\circ} \mathrm{C}$.

Siguiendo la metodología de Martínez et al. (6) con modificaciones, se inocularon en hoja suelta discos de micelio de $9 \mathrm{~mm}$ de diámetro obtenidos a partir de los bordes de las colonias de los aislados. Las mismas fueron dispuestas con la cara adaxial hacia arriba en bandejas de plástico transparente con tapa de 26×20x8 cm, sobre papel previamente esterilizado y humedecido con agua estéril.

Las inoculaciones del patógeno se realizaron mediante pulverización de suspensión de $10^{6}$ conidios $\mathrm{mL}^{-1}$ con el agregado Tween 20 , sobre plántulas de arroz (variedad GURI INTA CL) de 3-4 hojas desarrolladas en invernáculo.

Las plántulas inoculadas permanecieron en condiciones de cámaras húmedas durante el transcurso de la prueba de patogenicidad $(25 \pm$ $\left.2^{\circ} \mathrm{C}\right)(13)$.

Durante la campaña 2016/17, se recolectaron muestras de 48 hospedantes de Pyricularia spp. con síntomas de tizón: $O$. sativa 
(variedades Gurí INTA CL, Yerua, IC 110 y Carnaroli), Avena sativa, Bromus catharticus, Cenchrus cavanilliesii, Digitaria sp., Echinochloa colona, Eleusine indica, Eragrostis sp., Lolium perenne, Luziola peruviana, Pennisetum purpureum, Setaria italica, y S. sphacelata cv. Narok.

Las muestras de plantas analizadas presentaron una distribución estacional (Tabla 1). Las características morfométricas y culturales del patógeno coincidieron con las existentes en la bibliografía $(3,7,11)$.

E1 71\% de las muestras analizadas con síntomas, fueron halladas durante los meses de primavera y verano en plantas voluntarias, acompañando el ciclo del cultivo de arroz (excepto Cenchrus sp., Eragrostis sp. y Pennisetum sp.). Esta situación resalta la importancia de la presencia de otros hospedantes como fuente de inóculo potencial de la enfermedad en el arroz, aunque las relaciones patogénicas entre el cultivo y otros hospedantes, son poco frecuentes (11).

En $O$. sativa, las lesiones más avanzadas en la lámina foliar son fusiformes a romboidales, mientras que en las más jóvenes y esporulantes son de color verde más intenso. Lesiones más viejas, son color castaño a gris oscuro, con o sin bordes castaños. Finalmente numerosas lesiones tienden a unirse, secando la hoja y posteriormente la vaina foliar. En ataques tardíos se produce el secado de la panoja, la cual se inicia con áreas necróticas en el nudo basal del raquis, normalmente en coincidencia con una mancha en la lígula de la misma hoja de similar o mayor extensión que en raquis (Figs. $1 \mathrm{~A}, \mathrm{~B}, \mathrm{C}$ ).

B. catharticus, produce lesiones romboidales de tamaño variable, de 1 a $3 \mathrm{~mm}$ de ancho y 6 a $19 \mathrm{~mm}$ de largo, de centro y bordes castaño delgados rodeadas de un halo clorótico, las cuales confluyen extendiéndose en la lámina foliar formando el tizón característico; se observan de otoño a primavera (Fig. 1 D).

En Digitaria sp., a diferencia de B. catharticus, las lesiones son circulares a fusiformes. El centro es castaño a gris oscuro, con pérdida del área foliar y halo grisáceo en las lesiones de mayor edad, y borde delgado rojizo a castaño con halo clorótico en las más jóvenes. Se observan de primavera a otoño, con mayor frecuencia en verano, cuándo las condiciones ambientales son favorables (Figs. $1 \mathrm{~J}, \mathrm{~K}$ ).
En E. indica, es común encontrar el patógeno atacando el cuello de las inflorescencia, como así también en hojas. En las láminas foliares, los síntomas son muy similares a los que presenta el tizón del arroz. Si bien el síntoma está localizado en la unión de la inflorescencia con el raquis, el área necrótica puede extenderse hacia cada una de las espigas que comprenden el verticilo (Fig.1 F).

En Setaria italica, las lesiones son circulares y alargadas, de color marrón, rodeadas por un pequeño halo clorótico (Fig. $1 \mathrm{H}$ ).

Asociada a la sintomatología descripta, se observó la presencia del agente causal formando una eflorescencia grisácea constituida por conidióforos y conidios (Figs. $1 \mathrm{~L}, \mathrm{M}, \mathrm{N}$ ).

Del total de muestras analizadas, se obtuvieron 85 aislados de Pyricularia spp. Las características culturales de las colonias del hongo procedentes de los diferentes hospedantes, presentaron variaciones en cuanto al crecimiento, aspecto del micelio y colores (blanquecinos o crema, pasando por gris, hasta oliva oscuro) coincidiendo con las observaciones detalladas por Ou (7).

Los conidios de Pyricularia spp. presentaron variaciones en cuanto al tamaño y forma, entre distintos hospedantes, medio de cultivo e incluso entre diferentes órganos del mismo individuo. El hongo forma conidióforos simples que llevan en su extremo de 12 a 22 conidios hialinos, piriformes de base redondeada a obclavada y ápice reducido, con 2 septos, ligeramente constreñidos o no, de 17,1 (13,9-20,4) x 6,2 $(5,0-7,6) \mu$. Se observó que los conidios germinaron desde la célula apical o basal y con menos frecuencia, desde la célula media.

La patogenicidad fue comprobada en aislados de Digitaria sp. sobre Paspalum sp., S. italica sobre A. compresus, B. catharticus sobre E. indica (Figura 1, L) y $O$. sativa var GURI INTA CL sobre $O$. sativa var YERUA, utilizando el método de la hoja suelta, manifestando síntomas a los 7 días. En tanto que las inoculación realizadas con suspensión de conidios, dieron resultados positivos con aislados de $B$. catharticus sobre $O$. sativa var GURI INTA CL y $O$. sativa var GURI INTA CL sobre el mismo hospedante, cuyos síntomas fueron observados a los 8 días. Estas pruebas de patogenicidad, permitieron reproducir los síntomas de la enfermedad en las mencionadas combinaciones. No

Tabla 1. Hospedantes de Pyricularia spp y distribución estacional.

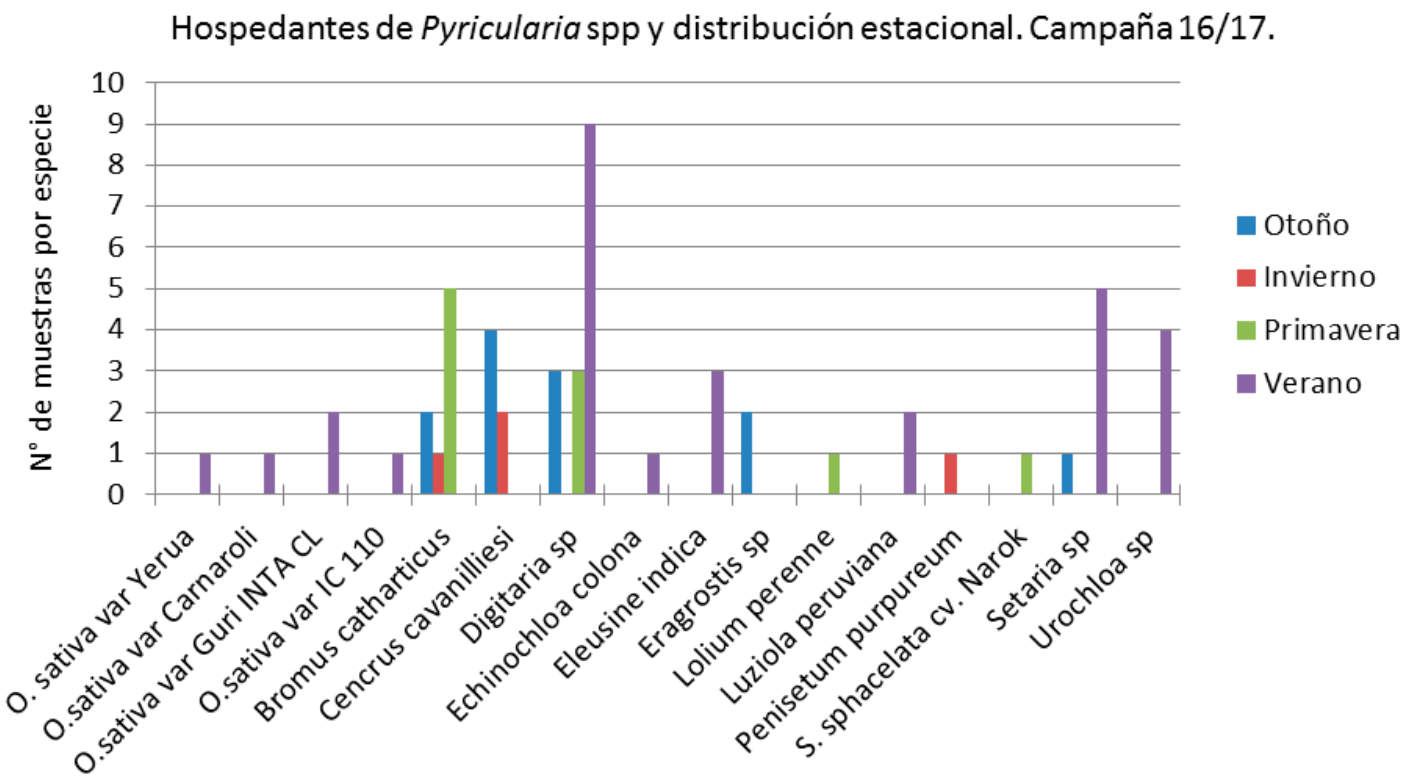

Hospedantes 


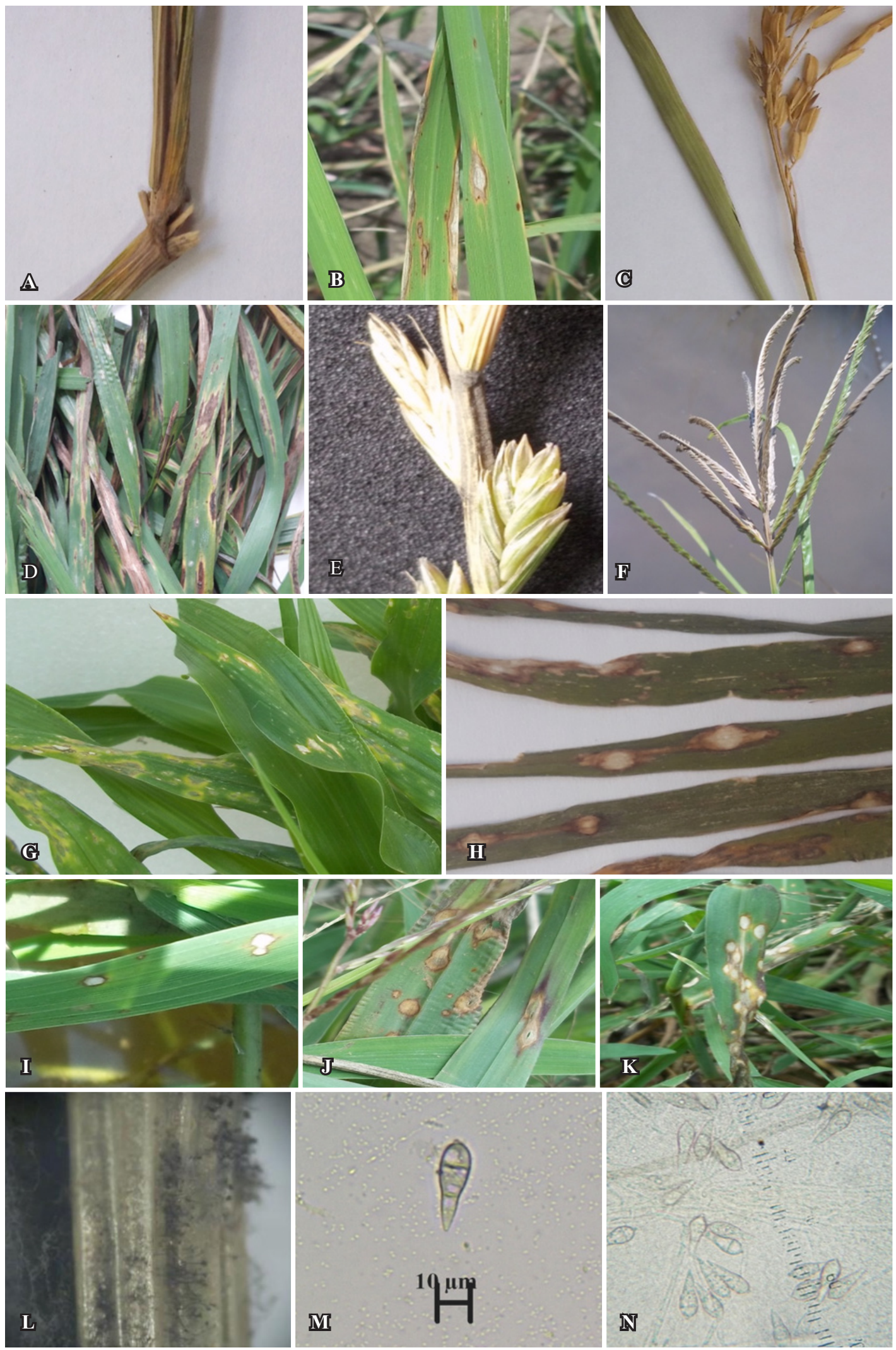

Figura 1.Síntomas causados por Pyricularia spp. Lesiones en condiciones de infección natural en lígula, lámina foliar y cuello de panoja de O. sativa var. GURÍ INTA CL (A, B, C), lámina foliar de B. catharticus (D), Urochloa sp. (G), S. italica (H), L. peruviana (I) y Digitaria sp. (J, $\mathrm{K}$ ), raquis de $L$. perenne (E) y E. indica (F). Micelio, conidios y conidióforos en cuello de nflorescencia de $E$. indica inoculado con aislado de $B$. catharticus (L), Conidio de aislado de A. sativa (M), medición de conidios en sustrato natural de $O$. sativa var. GURI INTA CL 40x factor de conversión $2,8(\mathrm{~N})$. 
obstante, se continúan con las inoculaciones directas y cruzada entre aislados obtenidos de malezas y arroz para confirmar su importancia como fuente de inóculo y el rango de virulencia de los aislados.

\section{AGRADECIMIENTOS}

A mis directores de beca doctoral: Dra. Gutierrez y Dr. Carmona por el apoyo constante y al directorio del CONICET por otorgamiento de la mencionada beca.

\section{REFERENCIAS}

1. Castroagudín, V.L.; Moreira, S.I.; Pereira, D.A.S.; Moreira, S.S.; Brunner, P.C.; Maciel, J.L.N.; Crous, P.W.; McDonald, B.A.; Alves, E.; Ceresini, P.C. Pyricularia graminis-tritici, a new Pyricularia species causing wheat blast. Persoonia, São Paulo, v.37, p.199-216, 2006. Disponível em: <https://www. ncbi.nlm.nih.gov/pubmed/28232765>. Acesso em: 10 dic. 2018.

2. Couch, B.C.; Kohn, L.M. A multilocus gene genealogy concordant with host preference indicates segregation of a new species, Magnaporthe oryzae from M. grisea. Mycologia, Mississauga, v.94, n.4, p.683-693, 2002.

3. Ellis, M.B. Dematiaceous Hyphomycetes. Kew Surrey: Commonwealth, 1971. 608p.

4. Goulart, A.P.; Amabili, R.F.; Nasser, L.C.B.; Freitas, M.A. Detecção de Pyricularia grisea em sementes de cevada produzidas em sistema irrigado por pivô central no cerrado brasileiro. Fitopatologia Brasileira, Brasilia, v.28, n.5, p.566, 2003. Disponível em: <http://www.scielo.br/pdf/fb/ v28n5/17676.pdf $>$. Acesso em: 10 dic. 2018.

5. Gutiérrez, S.A.; Cúndom, M.A. Pyricularia oryzae en cultivos de cebada en Corrientes (Argentina). Summa Phytopathologica, Botucatu, v.41, n.4, p.318-320, 2015. Disponível em: <http://www.scielo.br/pdf/sp/v41n4/01005405-sp-41-4-0318.pdf>. Acesso em: 1 dic. 2018.

6. Martínez, B.; Bernal, A.; Pérez, S.; Muñiz, Y. Variabilidad patogénica de aislamientos de Alternaria solani Sor. Revista de Protección Vegetal, La Habana, v.17, n.1, p.45-53, 2002. Disponível em: < https://www.research-
gate.net/publication/52012947_Variabilidad_patogenica_de_aislamientos_en_Alternaria_solani_Sor $>$. Acesso em: 10 dic. 2018 .

7. Ou, S.H. Rice diseases. 2 nd. ed. Kew Surrey: Commonwealth Mycological Institute, 1985. 380p.

8. Pedraza, M.V.; Liberman, C.A.; Asselborn, M.N. Pyricularia grisea en alpiste (Phalaris canariensis L.) en Argentina. In: Congreso Argentino de Fitopatología, 1., 2008, Córdoba. Libro de resúmenes. Córdoba: Asociación Argentina de Fitopatólogos, 2008. p.68. Disponível em: <http:// www.aafitopatologos.com.ar/media/secciones/416_desc.pdf $>$. Acesso em: 10 dic. 2018.

9. Pedraza, M.V.; Liberman, C.; Nuñez Bordoy, E.; Asselborn, M. Hospedantes secundarios como fuente de inóculo potencial del Quemado del Arroz en Argentina. In: Congreso Argentino de Fitopatología, 3., 2014, San Miguel de Tucumán. Anales. San Miguel de Tucumán: Asociación Argentina de Fitopatólogos, 2014. p.281. Disponível em: $<$ http://aafitopatologos.com.ar/ media/secciones/241_desc.pdf?05a317>. Acesso em: 10 dic. 2018.

10. Perelló, A.E.; Martinez, I.; Sanabria, A.; Altamirano, R.; Sibole, J.V. Pathogenicity of isolates of Magnaporthe spp. from wheat and grasses infecting seedlings and mature wheat plants in Argentina. Plant Pathology, La Plata, v.66, p.1149-1161, 2017. Disponível em: <http://onlinelibrary.wiley.com/ doi/10.1111/ppa.12658/full>. Acesso em: 10 dic. 2018.

11. Scheuermann, K.K.; Raimondi, J.V.; Marschalek, R.; Wickert, A.A.; Wickert, E. Magnaporthe oryzae Genetic Diversity and Its Outcomes on the Search for Durable Resistance. In: Caliskan, M. The Molecular Basis of Plant Genetic Diversity. Rijeka: InTech Europa, 2012. cap.15, p.331-356. Disponível em: $<$ https://www.intechopen.com/books/the-molecular-basis -of-plant-genetic-diversity/magnaporthe-oryzae-genetic-diversity-and-its -outcomes-on-the-search-for-durable-resistance>. Acesso em: 10 dic. 2018.

12. Tosa, P.; Chuma, I. Classification and parasitic specialization of blast fungi. Journal of Plant Pathology, Kobe, v.80, n.3, p.202-209, 2014. Disponível em: <https://link.springer.com/article/10.1007/s10327-014-0513-7>. Acesso em: 10 dic. 2018.

13. Verzignassi, J.R.; Poltronieri, L.S.; Benchimol, R.L.; Santos de França, S.K.; Arruda Carvalho, E.; Dornelas Fernandes, C. Pyricularia grisea: novo patógeno em Brachiaria brizantha cv. Marandu no Pará. Summa phytopathologica, Botucatu, v.38, n.3, p.254, 2012. Disponível em: <http://www scielo.br/scielo.php?script=sci_arttext\&pid=S0100-54052012000300016>. Acesso em: 10 dic. 2018. 